\title{
Reversing the weak measurement of an arbitrary field with finite photon number
}

\author{
Qingqing Sun, ${ }^{1, *}$ M. Al-Amri, ${ }^{2}$ and M. Suhail Zubairy ${ }^{1}$ \\ ${ }^{1}$ Department of Physics and Institute of Quantum Studies, Texas A\&M University, College Station, Texas 77843, USA \\ ${ }^{2}$ The National Centre for Mathematics and Physics, KACST, P.O. Box 6086, Riyadh 11442, Saudi Arabia \\ (Received 11 March 2009; revised manuscript received 17 July 2009; published 25 September 2009)
}

\begin{abstract}
For an arbitrary field with finite photon number inside a leaky cavity, we show that the null-result measurement can be conditionally reversed, using either multiple atoms or a multilevel atom. Even with photons detected, a certain class of fields can still be probabilistically recovered. The state protection probability in such a system can be significantly high.
\end{abstract}

DOI: $10.1103 /$ PhysRevA.80.033838

PACS number(s): 42.50.Dv, 03.65.Ta, 42.50.Pq

\section{INTRODUCTION}

In a quantum measurement, an operator projects the system into one of its eigenstates with a specific eigenvalue as the readout. Once the measurement is done, the unknown initial state of the system is destroyed, and in general there is no way to recover it from the result. However, such quantum measurement, the so-called strong or von Neumann measurement [1], is only part of the story. There is another type of quantum measurement called weak measurement [2], in which the outcome is not precise or sharp but nevertheless reveals some information about the system. Since this weak measurement does not totally collapse the system, the information of the initial state is passed over to the final state. If such retained information is complete, it would be possible to recover the initial state with some operations. This kind of state protection could be useful for quantum information processing.

One type of reversible measurements is deterministic, in which the initial state lies in a certain subspace and the measurement provides no information about it. Mabuchi and Zoller showed the conditions to unitarily invert quantum jumps in continuously monitored systems [3]. These conditions have been generalized by Nielsen and Caves to any ideal quantum operation [4], with quantum teleportation as a special example.

Another type is the probabilistically reversible measurements, for which only a certain outcome of the reversing measurement successfully restores the initial state. It has been discussed in quantum counter [5], quantum nondemolition measurement [6], and spin systems [7]. A general theory with necessary and sufficient conditions has been given in Ref. [8] as well as an information-theoretical analysis in Ref. [9]. Koashi and Ueda derived a trade-off relation between the unsharpness of the measurement and the best efficiency of the reversing operation [10]. In a recent experiment [11], based on a proposal by Korotkov and Jordan [12], the reversal of a weak measurement on a superconducting phase qubit was performed. A general procedure for $\mathrm{N}$-dimensional system was also proposed in [12], which requires $2^{N}$ steps.

An important question remains: can we reverse a multidimensional state in a simpler way? In Sec. II, we address this

\footnotetext{
*qsun@physics.tamu.edu
}

question and propose two schemes in cavity quantum electrodynamics (QED) systems, in which only a few steps are needed for the reversal. In Sec. III, we further consider clickallowed reversal for a specific class of states. Section IV is the conclusion.

\section{SCHEMES}

The state of interest is an arbitrary cavity field with finite photon number $\sum_{n, m=0}^{n_{\max }} \rho_{n m}|n\rangle\langle m|$, which is continuously monitored by an ideal photon detector outside. If there is no click, the field evolves into $\sum_{n, m=0}^{n_{\max }} \rho_{n m} e^{-(n+m) \gamma t}|n\rangle\langle m|$ according to the quantum trajectory theory, where $2 \gamma$ is the photon decay rate in the cavity and $t$ is the duration of the measurement. Here we ignore all the normalization constants for simplicity until the end of the derivation. For finite time this measurement is not sharp since any Fock component could give null result. To reverse the weak measurement, we need to swap the components symmetrically to $\sum_{n, m=0}^{n_{\max }} \rho_{n m} e^{-(n+m) \gamma t}|N-n\rangle\langle N-m|$, where $N\left(\geq n_{\max }\right)$ is an adjustable system parameter. Then we make another measurement. If it successfully produces null result again, the resulting field is $\sum_{n, m=0}^{n_{\max }} \rho_{n m} e^{-2 N \gamma t}|N-n\rangle\langle N-m|$. The common factor $e^{-2 N \gamma t}$ is dropped after normalization. Finally we swap back the components and the initial field is restored.

So the main challenge is how to swap the components $|n\rangle \rightarrow|N-n\rangle$ for all $n \in\left[0, n_{\max }\right]$ simultaneously. In our schemes, we realize it by using either multiple atoms or an atom with degenerate sublevels. Both schemes adopt the adiabatic passages to map the field coherence into the atoms, then swap the atomic levels with the help of some auxiliary fields and finally map the coherence back to the field. The swapping procedures for each scheme go as follows.

\section{A. Multiatom scheme}

The atoms are $\Lambda$ type with an excited level $|a\rangle$ and two lower levels $|b\rangle$ and $|c\rangle$. We prepare $N\left(\geq n_{\max }\right)$ atoms in level $|c\rangle$ and send them into the cavity. The diagram of the scheme is shown in Fig. 1. Two classical fields transversely propagate through two sides of the cavity. They are both resonant to the transition $|b\rangle \leftrightarrow|a\rangle$ and have space-dependent Rabi frequencies $\Omega(z)$ and $\Omega^{\prime}(z)$, where $z$ is the longitudinal coordinate. The cavity mode in the middle is resonant to the transition $|c\rangle \leftrightarrow|a\rangle$ with a coupling constant $g(z)$. To verify that 


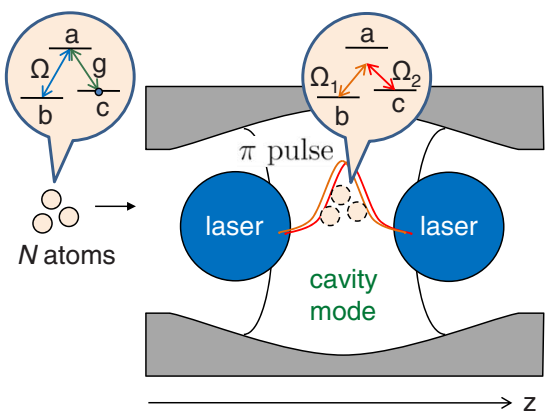

FIG. 1. (Color online) The multiatom scheme: an adiabatic passage maps the field state into the atoms. Then the atomic levels are swapped by an effective $\pi$ pulse. Finally another adiabatic passage maps the state back to the cavity field.

the scheme works, we consider a Fock component $|n\rangle$ of the cavity field.

Step (I): at the entrance the atoms encounter first the classical field $\Omega(z)$ and then the cavity mode $g(z)$. The Hamiltonian of the system can be written as [13]

$$
H=\sum_{i=1}^{N}\left(\hbar \Omega\left|a_{i}\right\rangle\left\langle b_{i}|+\hbar g| a_{i}\right\rangle\left\langle c_{i}\right| \hat{a}\right)+\text { H.c. },
$$

where $\hat{a}$ is the annihilation operator for the cavity mode. Such a system has a manifold of dark states [14], which evolve in parallel. Each number of excitations, either in the form of cavity mode photons or level $|b\rangle$ atoms, has its own dark state. The one with $n(\leq N)$ excitations is

$$
\begin{aligned}
\left|\Psi_{n}^{\mathrm{dark}}\right\rangle= & (-\Omega / g)^{n}|c \cdots c\rangle|n\rangle / \sqrt{n !} \\
& +(-\Omega / g)^{n-1} \sum_{i=1}^{N}\left|c \cdots b_{i} \cdots c\right\rangle|n-1\rangle / \sqrt{(n-1) !}+\cdots \\
& +\sum_{1 \leq i_{1}<\cdots<i_{n} \leq N}\left|c \cdots b_{i_{1}} \cdots b_{i_{n}} \cdots c\right\rangle|0\rangle .
\end{aligned}
$$

The key to state mapping is the adiabatic passage within the dark states. When the atoms move from the classical field region into the cavity mode region, they feel the adiabatic change from $\Omega \gg g$ to $\Omega \ll g$. The component evolves as

$$
|c \cdots c\rangle|n\rangle \rightarrow \sum_{1 \leq i_{1}<\cdots<i_{n} \leq N}\left|c \cdots b_{i_{1}} \cdots b_{i_{n}} \cdots c\right\rangle|0\rangle .
$$

Phase factor from $(-\Omega / g)^{n}$ is neglected here because it will be cancelled after the reversal.

Step (II): we add two other classical fields to the middle of the cavity. They have the same detuning $\nu_{1}-\omega_{a b}=\nu_{2}$ $-\omega_{a c}=\Delta$ and same Rabi frequency $\Omega_{1}=\Omega_{2} \ll \Delta$. Under the initial condition $c_{a}(0) \approx 0$, the excited level is adiabatically eliminated and each atom effectively behaves like a twolevel atom. The two lower levels swap with each other after a $\pi$ pulse, $|b\rangle \leftrightarrow-|c\rangle$. So the component changes as (a)

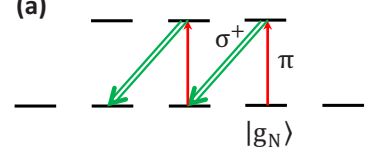

(b)

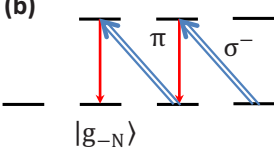

(c)

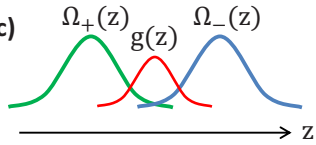

(d)

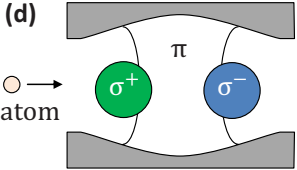

FIG. 2. (Color online) The Zeeman-level scheme: (a) level structure in step (i). The classical field is $\sigma^{+}$polarized and the cavity mode is $\pi$ polarized. (b) Level structure in step (II). The classical field is $\sigma^{-}$polarized. (c) The profiles of the fields required for the adiabatic passages. (d) The system diagram.

$$
\begin{aligned}
& \sum_{1 \leq i_{1}<\cdots<i_{n} \leq N}\left|c \cdots b_{i_{1}} \cdots b_{i_{n}} \cdots c\right\rangle|0\rangle \\
& \quad \rightarrow \sum_{1 \leq i_{1}<\cdots<i_{N-n} \leq N}\left|b_{i_{1}} \cdots c \cdots c \cdots b_{i_{N-n}}\right\rangle|0\rangle,
\end{aligned}
$$

which is just another dark state with $N-n$ excitations.

Step (III): now the atoms leave the cavity mode and encounter the other resonant classical field $\Omega^{\prime}(z)$. They feel the adiabatic change from $\Omega^{\prime} \ll g$ to $\Omega^{\prime} \gg g$, the dark states evolve as the reversal of Eq. (3):

$$
\sum_{1 \leq i_{1}<\cdots<i_{N-n} \leq N}\left|b_{i_{1}} \cdots c \cdots b_{i_{N-n}}\right\rangle|0\rangle \rightarrow|c \cdots c\rangle|N-n\rangle .
$$

So after the atoms fly out, the coherence is mapped back to the field, and the swapping $|n\rangle \rightarrow|N-n\rangle$ is achieved.

\section{B. Zeeman-level scheme}

The second scheme uses a degenerate two-level atom with $J_{g}=N+1 \leftrightarrow J_{e}=N$. We send in the atom prepared in the level $\left|g_{N}\right\rangle$. The diagram of the scheme is shown in Fig. 2(d). Two circularly polarized classical fields are placed at two sides of the cavity with the $\pi$ polarized cavity mode in the middle. They are all resonant to the atomic separation and the Rabi frequencies are $\Omega_{+}(z), \Omega_{-}(z)$, and $g(z)$, respectively. We examine the transformation of a cavity field component $|n\rangle$ below.

Step (I): the atom first encounters the $\sigma^{+}$field and then the cavity mode, with the level structure shown in Fig. 2(a). The dark state containing the component $\left|g_{N}\right\rangle|n\rangle$ is [15]

$$
\begin{aligned}
\left|\Psi_{n, g_{N}}^{\mathrm{dark}}\right\rangle= & \left|g_{N}\right\rangle|n\rangle \Omega_{N} \Omega_{N-1} \cdots \Omega_{N-n+1} \\
& +\left|g_{N-1}\right\rangle|n-1\rangle G_{N}^{(n)} \Omega_{N-1} \cdots \Omega_{N-n+1} \\
& +\cdots+\left|g_{N-n}\right\rangle|0\rangle G_{N}^{(n)} G_{N-1}^{(n)} \cdots G_{N-n+1}^{(n)},
\end{aligned}
$$

where $G_{k}^{(n)}=g(t) \sqrt{n+k-N}\left\langle J_{g}\left(m_{g}=k\right) ; 10 \mid J_{e}\left(m_{e}=k\right)\right\rangle$ and $\Omega_{k}$ $=\Omega_{+}(t)\left\langle J_{g}\left(m_{g}=k-1\right) ; 11 \mid J_{e}\left(m_{e}=k\right)\right\rangle$. For this configuration all the Clebsch-Gordan coefficients are nonzero. So when leaving the $\sigma^{+}$field and entering the cavity mode, the atom feels the adiabatic change from $\Omega_{+} \gg g$ to $\Omega_{+} \ll g$ and the state transforms as 


$$
\left|g_{N}\right\rangle|n\rangle \rightarrow\left|g_{N-n}\right\rangle|0\rangle,
$$

under the condition $n_{\max } \leq 2 N$. The atom jumps to the left sublevels, consuming one photon at each jump.

Step (II): in the latter half of the cavity, the atom only feels the cavity mode and the $\sigma^{-}$polarized field, as shown in Fig. 2(b). The new dark states are very similar to Eq. (6), except that now they connect all the ground levels to $\left|g_{-N}\right\rangle$. When the atom moves from the cavity mode to the $\sigma^{-}$field, it feels the change from $\Omega_{-} \ll g$ to $\Omega_{-} \gg g$. The adiabatic passage pumps the atom toward the left sublevels, generating one photon at each jump. In the end the state transforms as

$$
\left|g_{N-n}\right\rangle|0\rangle \rightarrow\left|g_{-N}\right\rangle|2 N-n\rangle \text {. }
$$

Then we remove the atom and the remaining field is $|2 N-n\rangle$.

We immediately find the advantage of this scheme: the fixed total number of jumps $(2 N)$ automatically swaps the components when we map the state back to the field, so only two steps are required instead of three as in the first scheme. Another advantage is that it does not require precise interaction time since there is no $\pi$ pulse.

\section{Probability and information analysis}

The reversing probability is just the probability of null result during the second measurement, which can be written as

$$
\begin{aligned}
P_{2}^{(0)}(t) & =\frac{\operatorname{Tr}\left(\sum_{n, m=0}^{n_{\max }} \rho_{n m} e^{-2 N \gamma t}|N-n\rangle\langle N-m|\right)}{\operatorname{Tr}\left(\sum_{n, m=0}^{n_{\max }} \rho_{n m} e^{-(n+m) \gamma t}|N-n\rangle\langle N-m|\right)} \\
& =\frac{e^{-2 N \gamma t}}{\sum_{n=0}^{n_{\max }} e^{-2 n \gamma t} \rho_{n n}}
\end{aligned}
$$

(or $N \rightarrow 2 N$ for the second scheme). In order to increase the success probability we should choose small $N$, down to the limit $N=n_{\max }$, in which case the probability is optimal because it reaches the upper bound $\min P^{(0)} / P^{(0)}(\rho)$ [12]. Since $e^{-2 N \gamma t}$ decreases faster than any term in the denominator, $P_{2}^{(0)}(t)$ decreases with time.

From the information-theoretical point of view, we can take the field being in state $|n\rangle$ as an event $x_{n} \in X$, and the detector reading of $k$ clicks as $y_{k} \in Y$. The mutual information gained from the first null-result measurement can be written as [16]

$$
I\left(X ; y_{0}\right)=\sum_{n=0}^{n_{\max }} p\left(x_{n} \mid y_{0}\right) \log \frac{p\left(x_{n} \mid y_{0}\right)}{p\left(x_{n}\right)},
$$

where $p\left(x_{n}\right)=\rho_{n n}$ is the initial probability and $p\left(x_{n} \mid y_{0}\right)$ $=e^{-2 n \gamma t} \rho_{n n} / \sum_{n^{\prime}=0}^{n_{\max }} e^{-2 n^{\prime} \gamma t} \rho_{n^{\prime} n^{\prime}}$ is the final probability for the field to be in $|n\rangle$. From Cauchy inequality we find $d I / d t$ $>0$ for all $t \in(0, \infty)$. The two limits are $I(0)=0$, which means no measurement (weakest), and $I(\infty)=-\log \rho_{00}$, which means a sharp measurement. So for a longer monitoring time, the measurement is stronger and more information is

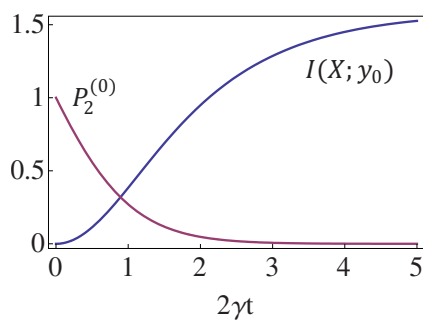

FIG. 3. (Color online) The reversal probability $P_{2}^{(0)}(t)$ and gained information $I\left(X ; y_{0}\right)$ for the initial state $(|0\rangle+|1\rangle+|2\rangle) / \sqrt{3}$. With the time passing by, more information is obtained while the chance of successful reversal diminishes.

gained, while the reversing probability decreases. We illustrate these trends with a simple example as shown in Fig. 3 .

\section{CLICK-ALLOWED REVERSAL}

So far we have been considering the reversibility of nullresult measurements. Such measurements do not destroy any component and only generate exponential factors which can be unified by a symmetric swapping and another null measurement. In the situation of photons being detected, the annihilation operator could destroy some components, and it also brings additional factors which may not be unified in general. Only a certain class of states, namely, the twocomponent states $\rho_{n n}|n\rangle\left\langle n\left|+\rho_{m m}\right| m\right\rangle\left\langle m\left|+\rho_{n m}\right| n\right\rangle\left\langle m\left|+\rho_{m n}\right| m\right\rangle$ $\langle n|$, can still be reversed probabilistically. If the photon detector registered $k(m>n \geq k)$ clicks during time $t$, the component $|n\rangle$ will change into

$$
e^{-\gamma \hat{a}^{\dagger} \hat{a} t} \hat{a}^{k}|n\rangle=e^{-\gamma(n-k) t} \sqrt{n ! /(n-k) !}|n-k\rangle,
$$

where we have ignored the prefactor $e^{-\gamma\left(t_{1}+\cdots+t_{k}\right)}$ because it will be cancelled after normalization. To reverse this measurement, we adopt the swapping procedures described in the two schemes. Taking $N$ (or $2 N$ in the second scheme) $=m$ $+n-k$, the component after swapping would be $e^{-\gamma(n-k) t} \sqrt{n ! /(n-k) !}|m\rangle$. After another measurement, provided there are $k$ clicks as well, we swap again and the component becomes

$$
e^{-\gamma(n-k) t} \sqrt{n ! /(n-k) !} e^{-\gamma(m-k) t} \sqrt{m ! /(m-k) !}|n\rangle .
$$

The initial $|m\rangle$ component will have the same factor which is symmetric to $m$ and $n$. So the normalized field is identical to the initial one.

The probability of $k$ clicks during the first measurement is

$$
P_{1}^{(k)}(t)=\left(1-e^{-2 \gamma t}\right)^{k}\left[\left(\begin{array}{l}
m \\
k
\end{array}\right) e^{-2 \gamma(m-k) t} \rho_{m m}+\left(\begin{array}{l}
n \\
k
\end{array}\right) e^{-2 \gamma(n-k) t} \rho_{n n}\right] .
$$

The physical meaning is clear: for a Fock state $|n\rangle$ to have $k$ clicks within time $t$, the photons have to be separated into two groups which causes the combination factor $\left(\begin{array}{l}n \\ k\end{array}\right)$. Each of the remaining $n-k$ photons contributes a factor $e^{-2 \gamma t}$, and each of the decayed $k$ photons contributes a factor $1-e^{-2 \gamma t}$. The reversing probability is the probability of emitting $k$ photons in the second measurement. Following the same argument, we find it to be 


$$
P_{2}^{(k)}(t)=\left(e^{2 \gamma t}-1\right)^{k} \frac{\left(\begin{array}{l}
m \\
k
\end{array}\right) e^{-2 \gamma m t}\left(\begin{array}{l}
n \\
k
\end{array}\right) e^{-2 \gamma n t}}{\left(\begin{array}{l}
m \\
k
\end{array}\right) e^{-2 \gamma m t} \rho_{m m}+\left(\begin{array}{l}
n \\
k
\end{array}\right) e^{-2 \gamma n t} \rho_{n n}} .
$$

When $k=0$, the above equation reduces to Eq. (9).

The advantage of click-allowed reversal is clear when we consider the probability of state protection. $P_{1}^{(k)}(t) P_{2}^{(k)}(t)$ gives the protection probability along the $k$-click path. So the total probability of protecting a field with two components $|m\rangle$ and $|n\rangle$ in time $2 t$ is

$$
P_{s}(2 t)=\sum_{k=0}^{n} P_{1}^{(k)}(t) P_{2}^{(k)}(t)=e^{-2 \gamma(m+n) t} \sum_{k=0}^{n}\left(e^{2 \gamma t}-1\right)^{2 k}\left(\begin{array}{l}
m \\
k
\end{array}\right)\left(\begin{array}{l}
n \\
k
\end{array}\right) .
$$

We can choose $n=m-1$ to optimize the result.

For the no-click reversal of a general state $\sum_{n, m=0}^{n_{\max }} \rho_{n m}|n\rangle\langle m|$, the probability of null result during the first measurement is $P_{1}^{(0)}(t)=\sum_{n=0}^{n_{\max }} e^{-2 n \gamma t} \rho_{n n}$. Therefore the protection probability is

$$
P_{s}^{(0)}(2 t)=P_{1}^{(0)}(t) P_{2}^{(0)}(t)=e^{-2 N \gamma t},
$$

which is highest when we take $N=n_{\max }=1$. Interestingly, both $P_{s}(2 t)$ and $P_{s}^{(0)}(2 t)$ are independent of the initial coefficients. Their comparison is shown in Fig. 4. The no-click reversal has higher protection probability for short time, while the click-allowed reversal is more successful for long time protection. This trend is more obvious for larger $m$ as those curves are more extended in the time domain. For a specific time we can find out which $m$ gives the best probability, as shown in Fig. 4 (inset). Even for $2 \gamma t=5$ when the no-click reversal gives a protection probability of only 0.0067 , the click-allowed reversal can still have a success chance above 0.2 although the $m$ required is around 100 which is quite a challenge. In case of a limited $m$, the longtime protection probability will be low. But we can increase it by split the time into many shorter cycles of measurement and reversal.

\section{CONCLUSION}

In summary, we propose two schemes to reverse the weak measurement of arbitrary states with finite photons. Taking advantage of the adiabatic passage in the dark states, the

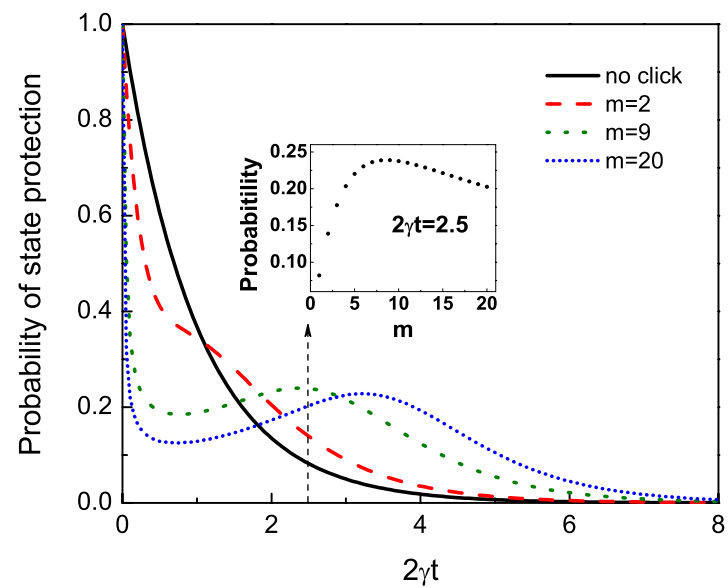

FIG. 4. (Color online) The state protection probability-time curves for no-click reversal, and click-allowed reversals with $m$ $=2,9,20(n \equiv m-1)$. (Inset) The probability- $m$ relation for a fixed $2 \gamma t=2.5$.

reversal can be achieved in a few simple steps. We also consider the click-allowed reversal for a certain class of states and show its advantage in state protection.

In all the derivations above, we assumed that the photon decay during the swapping procedures is negligible. This requires the adiabatic passage to be much faster than the typical photon decay time, i.e., $1 / T \gg \gamma$. The condition for adiabatic passage $g, \Omega \gg 1 / T$ also needs to be satisfied. The atomic decay can be neglected because the excited levels are never significantly populated. Another issue is the overall phase of the state, which may not be reversible. It could have an effect for applications such as interferometry.

The implementation of the first scheme could benefit a lot from the fact that experimentalists have been playing with $\Lambda$-type three-level atoms for a long time. For the second scheme, the degenerate two-level atom has many possible candidates, such as the transition $2 p^{5} 3 s\left({ }^{3} P_{2}\right) \rightarrow 2 p^{5} 3 p\left({ }^{3} P_{1}\right)$ in neon [17] and $1 s_{5}(J=2) \rightarrow 2 p_{10}(J=1)$ in argon [18]. There are also several techniques to prepare the atom into a Zeeman sublevel, see Ref. [19] and the references therein.

Note added: Recently, a paper on reversing the weak measurement for a photonic qubit using linear optics [20] has appeared.

\section{ACKNOWLEDGMENT}

This work was supported by a grant from the King Abdul Aziz City for Science and Technology (KACST).
[1] J. von Neumann, Mathematical Foundations of Quantum Mechanics (Princeton University Press, Princeton, 1955).

[2] A. N. Korotkov, Phys. Rev. B 60, 5737 (1999).

[3] H. Mabuchi and P. Zoller, Phys. Rev. Lett. 76, 3108 (1996).

[4] M. A. Nielsen and C. M. Caves, Phys. Rev. A 55, 2547 (1997).

[5] M. Ueda and M. Kitagawa, Phys. Rev. Lett. 68, 3424 (1992).
[6] A. Imamō̄lu, Phys. Rev. A 47, R4577 (1993).

[7] A. Royer, Phys. Rev. Lett. 73, 913 (1994).

[8] M. Ueda, N. Imoto, and H. Nagaoka, Phys. Rev. A 53, 3808 (1996).

[9] M. Ban, J. Phys. A 34, 9669 (2001).

[10] M. Koashi and M. Ueda, Phys. Rev. Lett. 82, 2598 (1999). 
[11] N. Katz, M. Neeley, M. Ansmann, R. C. Bialczak, M. Hofheinz, E. Lucero, A. O'Connell, H. Wang, A. N. Cleland, J. M. Martinis, and A. N. Korotkov, Phys. Rev. Lett. 101, 200401 (2008).

[12] A. N. Korotkov and A. N. Jordan, Phys. Rev. Lett. 97, 166805 (2006).

[13] M. O. Scully and M. S. Zubairy, Quantum Optics (Cambridge University Press, New York, 1997).

[14] T. Di, A. Muthukrishnan, M. O. Scully, and M. S. Zubairy, Phys. Rev. A 71, 062308 (2005).

[15] A. S. Parkins, P. Marte, P. Zoller, and H. J. Kimble, Phys. Rev.
Lett. 71, 3095 (1993); A. S. Parkins, P. Marte, P. Zoller, O. Carnal, and H. J. Kimble, Phys. Rev. A 51, 1578 (1995).

[16] S. Stenholm and K.-A. Suominen, Quantum Approach to Informatics (Wiley \& Sons, Hoboken, 2005).

[17] J. Martin, B. W. Shore, and K. Bergmann, Phys. Rev. A 54, 1556 (1996).

[18] W. Lange and H. J. Kimble, Phys. Rev. A 61, 063817 (2000).

[19] B. Wang, Y. Han, J. Xiao, X. Yang, C. Zhang, H. Wang, M. Xiao, and K. Peng, Phys. Rev. A 75, 051801(R) (2007).

[20] Y.-S. Kim, Y.-W. Cho, Y.-S. Ra, and Y.-H. Kim, Opt. Express 17, 11978 (2009). 